\section{$\underset{\substack{\text { hommes } \\ \text { \& migrations }}}{ }$}

\section{Hommes \& migrations}

Revue française de référence sur les dynamiques

migratoires

\section{$1320 \mid 2018$}

Au prisme de la consommation

\title{
Black Beauty
}

Jeux de frontières, mises en scène de soi et cosmopolitisme par le bas à Paris et Berlin

\section{Virginie Silhouette-Dercourt}

\section{(2) OpenEdition}

Journals

Édition électronique

URL : http://journals.openedition.org/hommesmigrations/4059

DOI : 10.4000/hommesmigrations.4059

ISSN : 2262-3353

Éditeur

Musée national de l'histoire de l'immigration

Édition imprimée

Date de publication : 1 janvier 2018

Pagination : 89-95

ISBN : 978-2-919040-40-7

ISSN : $1142-852 X$

Référence électronique

Virginie Silhouette-Dercourt, "Black Beauty », Hommes \& migrations [En ligne], 1320 | 2018, mis en ligne le 01 janvier 2021, consulté le 07 janvier 2021. URL : http://journals.openedition.org/ hommesmigrations/4059; DOI : https://doi.org/10.4000/hommesmigrations.4059 


\title{
BLACK BEAUTY \\ JEUX DE FRONTIÈRES, MISES EN SCÈNE DE SOI ET COSMOPOLITISME PAR LE BAS À PARIS ET BERLIN
}

Par VIRGINIE SILHOUETTE-DERCOURT, maître de conférences(HDR) en sciences de gestion, université Paris-13, CEPN, Centre Marc-Bloch, Berlin.

\author{
S'intéresser à la consommation de cosmétiques des femmes \\ noires et métissées à Paris et à Berlin, c'est plonger au cœur \\ de deux sociétés et de deux histoires migratoires, coloniales \\ et postcoloniales. C'est s'interroger sur la présence (ou non) de \\ modèles de beauté « de couleur » dans les médias nationaux, \\ sur la disponibilité (ou non) d'une offre commerciale dédiée à \\ cette beauté qui aurait pignon sur rue. En contrepoint, l'étude \\ des pratiques du quotidien des femmes concernées permet \\ d'analyser leurs mises en récit de soi. Aussi, la black beauty n'en \\ finit-elle pas de bousculer les marchés pour affirmer sa place \\ dans la ville et pour lutter contre les catégorisations sociales \\ dont elle fait l'objet.
}

Depuis une vingtaine d'années en France et en Allemagne se développent des marchés de cosmétiques « ethniques ». Basés sur l'hypothèse que les femmes noires et métissées ${ }^{1}$ ont dans ce domaine des besoins spécifiques dus à leur nature de peau et de cheveux, ces marchés sont fortement convoités par les entreprises ${ }^{2}$. Cette explication par la présence de besoins spécifiques laisse de côté d'autres ressorts plus classiquement mobilisés en sciences humaines et sociales lorsqu'il s'agit d'expliquer le recours aux cosmétiques. Des recherches, par exemple, établissent un lien entre l'utilisation de cosmétiques et l'estime de soi ${ }^{3}$. Une apparence physique idéale, c'est-à-dire répondant à des critères de beauté stéréotypés, détermine le succès interpersonnel et l'estime de soi. Or ces critères, qui sont devenus un véritable corset psychologique pour beaucoup de femmes ${ }^{4}$, sont dominés par les standards de beauté occidentaux - la minceur et la pâleur du teint par exemple ${ }^{5}$.

Depuis 2005, mes recherches portent sur le sens que donnent les jeunes femmes noires et métissées à leurs pratiques de beauté. À travers leurs récits de vie et l'observation de leurs rituels de beauté, je 
tente de saisir le rôle des soins de beauté dans la négociation des appartenances ${ }^{6}$. La consommation de cosmétiques fait clairement partie des pratiques transnationales, entre ici et là-bas ${ }^{7}$. Je montre que l'inventivité du quotidien qu'elles déploient en matière de beauté est l'un des moyens qu'elles mobilisent pour tenir à distance les déterminismes de classe, de race et de genre ${ }^{8}$. Ce travail sur la société française m’a amené à partir de 2010 à analyser d'autres contextes, notamment une autre société formée autour d'un autre rapport à la nationalité et d'une autre histoire migratoire : l'Allemagne. Je me suis intéressée là encore au rôle des cosmétiques et des lieux de consommation dans les parcours de femmes noires et métissées dans la ville-État de Berlin. J'ai interrogé majoritairement des mères de famille nées en Allemagne ou arrivées à l'âge adulte.

\section{Comparer l'incomparable?}

L'objectif de cette contribution est de retracer la façon dont, dans deux contextes nationaux différents et dans deux villes-mondes, Paris et Berlin" les cosmétiques et leur usage sont révélateurs de processus individuels et sociaux plus complexes. Les interactions sociales que ces femmes vivent dans le quotidien de ces deux villes influencent les mises en scène d'elles-mêmes qu'elles vont choisir d'endosser sans que cela soit toujours conscient ou intentionnel. On retrouve ici les vicissitudes de la présentation de soi décrites par Erving Goffman ${ }^{10}$. Malgré les différences entre les deux villes, les femmes interrogées déploient une forme de cosmopolitisme «par le bas » au sens d'Arjun Appadurai ${ }^{11}$ : un cosmopolitisme qui commence localement dans les pratiques du quotidien mais qui étend son réseau de solidarité vers le global, par le biais de filières diasporiques, afin de lutter contre l'exclusion et contre les catégorisations sociales dont elles font l'objet alors qu'elles sont bien souvent « françaises » ou « allemandes ».

Tant dans la structure de leurs populations que dans les pratiques de beauté, les deux capitales présentent des différences marquées. L'Allemagne a accueilli récemment un grand nombre de demandeurs d'asile ${ }^{12}$ et Berlin a vu la part de sa population avec un arrière-plan migratoire passer le seuil des $30 \%{ }^{13}$. Les groupes les plus représentés ne viennent cependant pas d'Afrique subsaharienne mais, principalement d'Europe, d'Asie, de Turquie ou de Pologne ${ }^{14}$. L'Allemagne reçoit finalement un petit nombre de personnes originaires du continent africain sur son territoire par rapport à d'autres groupes ${ }^{15}$ même si la crise migratoire récente change un peu la donne ${ }^{16}$. La France, de son côté,

6. Virginie Silhouette-Dercourt, « Consommer comme ici ou comme là-bas? Les dimensions identitaires de la consommation de cosmétiques des jeunes femmes issues de l'immigration subsaharienne en France ", in Hommes \& Migrations, $\mathrm{n}^{\circ}$ 1286-1287, 2010, pp. 190-198 ; Virginie Silhouette-Dercourt, "Les quartiers africains de Paris comme lieux d'inclusion et d'exclusion par la consommation ", in Hommes \& Migrations, $n^{\circ}$ 1308, 2014, pp. 97-103. 7. Le concept de transnationalisme a été forgé au début des années 1990 pour désigner l'ensemble des pratiques sociales, économiques, politiques à travers lesquelles les immigrés maintiennent des liens étroits avec leur pays d'origine. 8. Virginie Silhouette-Dercourt, Beauté ethnique sous tension. Entre marginalisation, injonctions républicaines et inventivité du quotidien, Paris, EMS, 2017. 9. Cette analyse prend appui sur une quarantaine d'entretiens : dont 22 menées en Île-de-France (93) auprès de jeunes femmes (18-25 ans) étudiantes nées en France de parents d'origine africaine; et à Berlin auprès de 18 femmes (24-62 ans), mères de famille, soit nées en Allemagne de parents originaires d’Afrique subsaharienne, soit arrivées en Allemagne à l'âge adulte. À Berlin, je tiens ici à remercier Hadja Kitagbe et l'association Mama Afrika qui luttent contre l'excision des jeunes filles en Guinée et en Allemagne. C'est grâce aux petits-déjeuners organisés tous les mois par cette association, ouverts aux femmes africaines vivant à Berlin, que j'ai pu mener une grande partie de mes entretiens en Allemagne. 10. Erving Goffman, La mise en scène de la vie quotidienne I, La présentation de soi, Paris, éd. de Minuit, 1973. 11. C'est-à-dire un activisme des populations les plus exclues. II prend ici l'exemple des bidonvilles de Bombay. Voir Arjun Appadurai, "Cosmopolitanism from below : some ethical lessons from the slums of Mumbai ", in The Johannesburg Workshop in Theory and Criticisms, $n^{\circ}$ 4, 2011, pp. 32-43. 12. 974551 demandeurs d'asile ont été accueillis en 2015 dont 308021 en provenance de Syrie. Source : https://www.destatis.de/DE/ZahlenFakten/GesellschaftStaat/ Soziales/Sozialleistungen/Asylbewerberleistungen/Tabellen/9_Empf_Staatsang_RegellstgGeschl.html;jsessionid=7073F35Ao66B47CB9 E9E45872B1A9A8C.cae4 13. En 2015. Source : https://www.statistik-berlin-brandenburg.de/BasisZeitreiheGrafik/Bas-Bevoelkerungsstand. asp Ptyp $=300 \&$ Sageb $=12015$ \&creg=BBB\&anzwer $=6$ 14. En 2016, sur la ville de Berlin, les naturalisations ont concerné principalement des personnes originaires de Turquie (936) et de Pologne (461). L'ensemble des naturalisations de personnes issues du continent africain s'élevait à 602, dont 90 originaires du Cameroun, 80 du Nigeria et 36 du Ghana. Url : https://www.statistik-berlin-brandenburg.de/ publikationen/stat_berichte/2017/SB_A01-09-00_2016j01_BE.pdf. 15. En 2007, un peu moins de 270 ooo ressortissants du continent africain étaient recensés. Source : Eurostat, Statistisches Bundesamt (Destatis). 16. Voir les chiffres de 2015 sur l'accueil de demandeurs d'asile issus de l'Érythrée, du Nigeria ou de Somalie. Url : https://www.destatis.de/DE/ZahlenFakten/GesellschaftStaat/Soziales/Sozialleistungen/ Asylbewerberleistungen/Tabellen/9_Empf_Staatsang_RegellstgGeschl.html;jsessionid=7073F35A066B47CB9E9E45872B1A9A8C.cae4. 
est un ancien pays d'immigration, une vague d'immigration succédant à une autre. Son passé colonial plus ancien explique en partie la présence d'une importante seconde génération d'immigration ${ }^{17}$ notamment afro-française à Paris et en Îlede-France. En effet, si près d'un tiers des immigrés et de leurs descendants vivent en Île-de-France ${ }^{18}$, cette part est multipliée par deux en ce qui concerne les descendants d'Afrique subsaharienne : les deux tiers sont, en effet, franciliens ${ }^{19}$. Ces chiffres ne prennent pas en compte les personnes issues de l'outre-mer. Ainsi des histoires migratoires différentes, coloniales et postcoloniales, ont engendré des structures de populations bien différentes dans les deux villes.

\section{Afro-Deutsch et Afro-Français}

Pourtant, en Allemagne et plus particulièrement à Berlin, l'immigration africaine est une histoire ancienne. Dès le XVIII siècle, Frédéric II, roi de Prusse, électeur de Brandebourg, fait venir des Africains à la cour ${ }^{20}$. À la fin du XIXe, l'Allemagne unifiée s'engage dans la concurrence coloniale sur le continent africain mais aussi dans le Pacifique et en $C_{\text {Chine }}{ }^{21}$. Des migrants issus de ces colonies viennent parfois s'installer en Allemagne durant cette période et, jusqu’à la Première Guerre mondiale, ils y développent une activité et fondent une famille. Après la guerre, une partie de la Rhénanie et de la Sarre est occupée par des troupes françaises composées de soldats issus de l'empire colonial français d'Afrique et d'Asie. Plusieurs centaines d'enfants afro-allemands seraient nés de cette première occupation. La période du nationalsocialisme est une période particulièrement tragique pour ces Afro-Allemands qui perdent leur nationalité, subissent des sévices et des stérilisations forcées, ou périssent en déportation. Après la guerre, entre 1945 et 1955, près de 400000 enfants appelés les enfants de l'Occupation ou Besatzungskinder naissent ${ }^{22}$. Puis, lors de la partition du pays, la migration d'origine africaine prend différentes formes entre l'Est et l'Ouest. À l'Ouest, les candidats au départ viennent pour faire des études, suivre une formation, demander l'asile ou dans le cadre du regroupement familial. Mais, à l'Est, des étudiants ou des contractuels sont acceptés sur une base temporaire avec impossibilité de pratiquer un regroupement familial. La communauté africaine vit séparée, dans des logements à lécart, avec peu de contact avec la société allemande. Malgré cette longue histoire commune, ce n'est qu'à partir des années 1980 que s'affirme véritablement une identité noire ou "Afro-Deutsch» en Allemagne, inspirée par le mouvement afro-américain et portée par May Ayim Opitz ${ }^{23}$. En France aussi, émerge avec le Conseil représentatif des associations noires de France (Cran), un mouvement visant à défendre

17. Gérard Bouvier indique que la première génération de personnes de $25-54$ ans nées à l'étranger représente $13,1 \%$ de l'ensemble des 25-54 ans en France, contre 17,6\% en Allemagne. En ce qui concerne la " deuxième génération ", ce chiffre serait de $13,5 \%$ pour la France et de 4,3\% pour l'Allemagne. L'ensemble " première et deuxième génération » représente donc 26,6 \% pour la France et 21,9\% pour l'Allemagne (p. 19). Chiffres de 2008. Voir Gérard Bouvier « Les descendants d'immigrés plus nombreux que les immigrés: une position française originale en Europe ", Paris, INSEE, 2012. Url : https://www.insee.fr/fr/statistiques/1374014? sommaire=1374025. 18. Voir Catherine Borrel, Bertrand Lhommeau, "Être né en France d'un parent immigré ", Paris, INSEE, $n^{\circ}$ 1287, 2010. 19. Certaines villes du Grand-Paris connaissent ainsi des concentrations très élevées comme dans le département de la Seine-Saint-Denis : Clichy-sous-Bois, Aubervilliers et La Courneuve - villes dans lesquelles environ trois-quarts des jeunes sont d'origine étrangère, $70 \%$ à Saint-Denis et $67 \%$ à Saint-Ouen mais aussi d'autres départements comme l'Essonne avec, par exemple, Grigny où $70 \%$ des jeunes sont d'origine étrangère. Voir Bernard Aubry, Michèle Tribalat, "Les concentrations ethniques en France : évolution 1968-2005 ", in Espaces populations et sociétés, $n^{\circ}$ 3, 2011, pp. 493-507. 20. En France, le Code noir réglemente dès la fin du XVII siècle la vie des esclaves vivant dans les îles de l’Amérique française. Cette histoire coloniale se prolonge et prend de l'ampleur pendant tout le XVIII siècle avec le commerce triangulaire, puis au milieu du XIX siècle avec la mise en place du Second Empire colonial. 21. À partir de 1884, le chancelier Otto Von Bismarck favorise l'installation de colonies et de comptoirs d'échanges. L'empire allemand se consolide peu à peu avec la Namibie (1884), le Cameroun et le Togo (1884), la Tanzanie et le Rwanda-Urundi (1885). Voir l'exposition consacrée récemment à cette histoire coloniale au Deutsches Historisches Museum de Berlin. http://www.dhm.de/ausstellungen/archiv/2016/deutscher-kolonialismus.html 22. Silke Satjukow, Rainer Gries, "Bankerte! "Besatzungskinder in Deutschland nach 1945, Francfort, Campus, 2015. Voir aussi, sur les violences sexuelles subies par les femmes allemandes à la fin de la guerre, Miriam Gebhardt, Als die Soldaten kamen. Die Vergewaltigung deutscher Frauen am Ende des Weiten Weltkriegs, Munich, DVA, 2015. 23. Theodor Wonja Michael, né en 1925 à Berlin d'un père camerounais et d'une mère allemande, raconte dans sa biographie l'importance pour la communauté afro-allemande de cette catégorie et de son pouvoir de mobilisation. II cite le livre Farbe Bekennen, Afro-deutsche Frauen auf den Spuren ihrer Geschichte, publié en 1986 par May Ayim Opitz. Voir Theodor Wonja Michael, Deutsch sein und schwarz dazu: Erinnerungen eines Afro-Deutschen, Munich, Deutscher Taschenbuch Verlag, 2013. 
Magasin de produits de coiffure afro, rue du Château-d'Eau (Paris Xe), novembre 2017. Photo Camille Millerand. @ Camille Millerand.

les droits des populations noires, à faire reconnaître leur place et les discriminations dont elles font l'objet ${ }^{24}$. Si les termes «Afro-Français » ou «AfroAllemands » recoupent des parcours et des réalités très différentes, ils sont cependant unis par des expériences communes de discrimination, de marginalisation et de stigmatisation.

\section{Différences de visibilité pour les lieux de consommation « ethnique »}

À Paris, se déploient depuis les années 1960 des centralités commerciales dédiées à la Black Beauty ${ }^{25}$, tandis qu'à Berlin, la visibilité ou la concentration de ces lieux est moindre ${ }^{26}$. Car ce sont d'abord des entrepreneurs turcs, italiens, grecs ou de l'exYougoslavie ${ }^{27}$ qui ont marqué la ville de leur présence par le biais de boutiques, cafés, kebabs ou restaurants qu'ils ont créés. Les femmes interrogées circulent entre les quartiers et les boutiques autour d'Osloerstrasse, de Wedding, ou de Neukoln. Dans le quartier "africain » (Afrikanisches Viertel ${ }^{28}$ ) du nom des rues qui le compose et situé au nord-ouest de la ville, on remarque dans certaines rues des restaurants, des boutiques ou des coiffeurs dédiés à la communauté afro-berlinoise, mais toujours de façon éparse. Les rues de ce quartier portent encore aujourd'hui les noms d'anciennes colonies et de personnalités controversées de cette histoire coloniale ${ }^{29}$. L'idée est de rompre avec ce passé en débaptisant certaines de ces rues pour valoriser des personnalités afro-allemandes.

24. Voir en France le Conseil représentatif des associations noires de France (Cran) créé en 2005, et, en Allemagne, Initiative Schwarz Menschen, créé en 1985. 25. Virginie Silhouette-Dercourt, "Les quartiers africains de Paris... ", op. cit. 26. Exemples de boutiques dédiées à la Black Beauty à Berlin : http://www.afrika-in-berlin.net/entries?cat=5 27. Voir, par exemple, Felicitas Hillmann, Migration: Eine Einführung aus sozialgeographischer Perspektive, (Sozialgeographie kompakt; Band 4), Stuttgart, Steiner, 2016 ; Andreas Ludwig, Christopher Neumaier, "Individualisierung der Lebenswelt. Konsum, Wohnkultur und Familienstrukturen ", in Frank Bösch (dir.), Geteilte Geschichte. Ost- und Westdeutschland 1970-2000, Göttingen, V\&R, 2015, pp. 239-282; Maren Möhring, " Döner Kebab and West German consumer (multi)cultures ", in Ulrike Lindner, Mark Stein, Silke Stroh (dir.), Hybrid Cultures, Nervous States. Insecurity and Anxiety in Britain and Germany in a (Post)Colonial World, Amsterdam/New York, Rodopi, 2011, pp. 151-165. 28. Partie du quartier de Wedding en forme de triangle entre les rues Müllerstraße, Seestraße et Afrikanische straße, composée des rues Togo, Cameroun, Sansibar, Guinée, Transvaal... 29. Comme les rues Lüderitzstraße ou Nachtigalplatz qui depuis fin 2016 doivent être rebaptisées. 
D'un point de vue économique, l'Allemagne est cependant un marché de cosmétiques plus important que la France et se classe au $5^{\mathrm{e}}$ rang mondial derrière les USA, le Japon, le Brésil et la Chine. En Europe, la maturité des marchés a conduit les industriels du secteur à s'intéresser à des segments précédemment considérés comme des «niches » comme celui des seniors, des produits naturels ou bios, et des consommateurs « ethniques ». Les offres ciblant ce dernier marché s'y développent très rapidement. Cependant, on remarque, à Paris comme à Berlin, que les produits à destination des femmes noires ou métissées ne se sont pas diffusés de la même manière. En Île-de-France, les principales enseignes comme Carrefour, Auchan ou Leclerc ont créé des rayons « ethniques » pour répondre à la diversité des attentes en termes d'alimentation. Mais l'offre de cosmétiques dédiés à une clientèle noire ou métissée peine à s'affirmer. Les distributeurs allemands se sont engagés encore plus timidement sur ces marchés. À Berlin, les supermarchés comme Edeka ou Kaiser proposent dans leurs rayons des aliments dédiés à une clientèle internationale et composés de plats orientaux, de soupes et nouilles chinoises, de boîtes de sushis. Cependant, le rayon cosmétique reste limité à une clientèle "caucasienne ". De même, les chaînes de distribution spécialisées dans l'hygiène et la beauté comme Dm-Drogerie Markt ou Rossmann n'ont pas créé au sein de leur offre classique des produits dédiés à ces beautés.

\section{Exprimer ses appartenances multiples, contourner les assignations}

À Berlin comme à Paris, les pratiques cosmétiques des femmes interrogées reflètent des questionnements sur une fabrication de soi où la consommation de produits cosmétiques joue un rôle important. Ceci est particulièrement prégnant pour les femmes nées en Europe de parents venant $\mathrm{du}$ continent africain et qui vivent une sorte d'entre-deux. Voici, par exemple, ce que dit
Bénédicte, jeune femme de 20 ans, née en France de parents originaires de la République démocratique du Congo ${ }^{30}$, étudiante à Paris : «Moi, je sais que je suis française, mais voilà je suis française d’origine zaïroise, je ne peux pas me permettre de dire que je suis une Française. C'est tout, je suis obligée de revenir sur mes origines. C'est pour ça que je ne peux pas me sentir totalement française. Mais aussi, je suis de culture zaïroise, en plus une culture qui n'est pas forcément acceptée, qui ressort pas... Je ne sais pas comment vous dire, en fait je suis française de papiers, j’ai une culture, deux cultures... » Ou Anne Shanna, femme de 48 ans, née à Berlin-Est de mère allemande et de père tanzanien : « Mais oui, je suis berlinoise, bien sûr ! Mais je me suis toujours sentie différente... À l'école jétais la seule... Les autres frottaient ma peau pour enlever la couleur... Ma mère ne savait pas comment démêler mes cheveux... »

Pour ces femmes nées en France ou en Allemagne, se pose la question des origines, dans leur quotidien et dans leurs interactions sociales, alors qu'elles sont bien souvent françaises ou allemandes. Ces assignations sont également très fortement ressen-

À Berlin comme à Paris, les pratiques cosmétiques des femmes interrogées reflètent des questionnements sur une fabrication de soi où la consommation de produits cosmétiques joue un rôle important.

ties lorsqu'elles cherchent des produits qui conviennent à leur type de peau ou de cheveux, ou des coiffeurs capables de les coiffer sans les abîmer.

Voici ce qu'explique Acha, mère de famille camerounaise de 25 ans (arrivée en Allemagne à 18 ans) qui a du mal à trouver à Berlin des coiffeurs adaptés pour coiffer les cheveux de sa fille : "J'ai essayé des coiffeurs ici. Ils ne connaissent pas le cheveu africain. Alors je me débrouille avec les amies, les cousines, les tantes... » Le fait de ne pas exister sur les marchés, en termes d'offres de produits et de services (coiffeurs), les renvoie à leur différence. Dans le quotidien de celles qui ont été élevées et éduquées en France ou en Allemagne, ne connaissant pas ou peu leurs pays d'origine, le rappel constant de leurs origines - notamment dans le contexte berlinois où elles sont numériquement très minoritaires - les amènent à puiser dans un réservoir de pratiques, 
de produits ou d'ingrédients issus d'un pays d'origine qu'elles recréent ou qu'elles fantasment, sorte de refuge durant les temps difficiles.

D'ailleurs, à Berlin, certaines mères mettent en place des stratégies pour entretenir ce refuge et la fierté associée. C'est le cas de Fatoumata, femme de 48 ans née en Guinée, arrivée à Berlin il y a 20 ans et qui a envoyé chacun de ses quatre enfants au pays : "Moi, je suis guinéenne de naissance, je le reste, j'essaye de transmettre cela à mes enfants (....) Avec mon mari, on était d'accord là-dessus : chacun de nos enfants est allé au pays vivre une année scolaire, pour qu’il soit fier de ses origines. Au début, ça a été très dur pour eux mais après, ils ne voulaient plus rentrer à Berlin. » Cette transmission passe également par le rapport au corps et à la beauté : "Quand ils sont làbas, ils voient tous ces coiffeurs pas chers et qui savent te coiffer, ils voient la mode africaine, les couleurs... » Pour d'autres, la recherche de cosmétiques associés au pays d'origine permet de trouver du réconfort dans des moments de passage à vide comme l'explique Mouneissa, mère ivoirienne de 45 ans, arrivée à Berlin il y a cinq ans : "L'hiver, c'est très dur, pas de lumière. J'ai la peau, les cheveux très secs. Dehors, il fait froid, alors je prends une douche chaude, chaude, chaude, et je m'enduis de beurre de karité. Je reste des heures comme ça sous la douche. Après, ça va mieux. C'est comme un médicament, un médicament de l'âme en fait [rires]. »

Pour les femmes vivant à Berlin, Paris est comme un pont entre l'Allemagne et l'Afrique, comme l'exprime Acha : «Paris, c'est presque l'Afrique hein! » Elles vont dans les quartiers autour des stations de métro Château-d'Eau ou Château-Rouge et y retrouvent de la famille.

\section{Se mettre en scène en fonction des contextes sociaux}

Les femmes interrogées adaptent leur discours et leur façon de se présenter aux autres en fonction du contexte social dans lequel elles évoluent. Par exemple, Hadja change sa présentation en termes de maquillage, de coiffure, de vêtement, en fonction du contexte et de ses interlocuteurs. Lors de nos entretiens, elle se présentait en pantalon jeans, non maquillée et les cheveux naturels. Mais lors de présentations officielles de son association, elle endossait un autre rôle et un autre personnage : elle adoptait une coiffure africaine avec des tresses et des perles, son maquillage des yeux et des lèvres était visible et elle portait le costume traditionnel africain. Elle échangeait de préférence en français, quitte à faire appel à un(e) interprète. Devant les membres de la communauté afro-berlinoise, elle s'adressait davantage en malinké et portait également la coiffe et le costume traditionnels, quoique dans une version plus simple et plus modeste. Selon la façon dont elle se présentait aux autres, cherchait-elle à contrôler les termes de l'interaction sociale et les réactions des personnes impliquées dans l'échange ${ }^{31}$ ?

D'autres femmes interrogées mettent en scène différents « visages » d'elles-mêmes en distinguant différents contextes sociaux ou relations interpersonnelles, comme des caméléons. C'est le cas d'Anne Shanna en arrivant à Paris : «À Berlin, je me sentais petite et noire. À Paris, tout d'un coup, comme par un coup de baguette magique, je me suis sentie grande et claire... Avec tous ces Maghrébins et ces Africains, je me sentais claire, j'avais cet accent allemand. Donc on voyait bien que je rétais pas Africaine, que j'étais Européenne. On me demandait tout le temps: "Mais d'où tu viens ?" Le fait que je sois moitié allemande et que mes cheveux soient défrisés me situait ailleurs que parmi les Africains... »

\section{Un cosmopolitisme par le bas à Paris et à Berlin}

Avant l'arrivée d'Internet et des sites de commerce en ligne, la faible présence d'une offre dédiée à la Black Beauty a entraîné la création, de la part de certaines femmes rencontrées, de petits commerces dédiés à la beauté. Comme pour le commerce alimentaire, ces entrepreneuses migrantes n'avaient 
pas de formation particulière dans ce domaine mais se sont résolues à créer des entreprises du fait de l'absence d'offre. Fatima (mère guinéenne, arrivée à 20 ans à Berlin) raconte : "J'ai ouvert cette boutique, j'allais à Paris en voiture acheter des stocks que je revendais à Berlin, il n'y avait rien à l'époque... »

D'autres, comme Sharon, 20 ans, née au Cameroun et arrivée à Paris à 5 ans, ramènent les produits du pays : "Quand je reviens du Cameroun, je ramène toujours plein de choses : du beurre de karité, le stick Silver Rose (contre les boutons), mais aussi des mèches et extensions... »C'est ici un exemple de pratique transnationale au sens d'Alejandro Portes, c'est-à-dire : " une construction de champs sociaux dans laquelle les migrants créent un lien - imaginaire ou réel - entre le pays d'origine et la société d'accueil ${ }^{32}$. »

Parfois, il suffit d'appeler une lointaine cousine au Canada ou aux États-Unis pour se faire livrer la nuance de fond de teint adaptée. L'arrivée d'Internet et du commerce en ligne a accéléré ces échanges qui portent sur les produits mais aussi sur les conseils de coiffage, de maquillage, les recommandations de nouveaux produits ou de nouvelles coupes à la mode comme l'explique Mariam, 22 ans, née à Paris de parents de nationalité sénégalaise: "Je me renseigne d'abord sur Internet, il y a plein de bloggeuses qui parlent des produits, des coupes. » À Berlin, l'Internet a permis l'accès à des produits américains qu'il était difficile de se procurer. L'activisme de ces femmes a ainsi modifié les quartiers, les commerces et, dans le cas de Paris - davantage qu'à Berlin -, l'offre de produits disponibles dans la grande distribution. À Paris, au lieu d'être cantonnés dans des zones et points de vente "ethniques », les produits, ingrédients et rituels de beauté se sont, en effet, diffusés dans les circuits «classiques » - nouveautés incluant du beurre de karité, rayons dédiés à ces produits - et sont devenus des sources d'inspiration pour les industriels du secteur : développement de gammes, lancement de nouvelles marques, de nouvelles propositions de coiffage... On peut ainsi ici parler d'un véritable cosmopolitisme par le bas $^{33}$ qui renouvelle les marchés. En effet, à travers des initiatives comme la création de commerce, les liens et échanges avec la famille restée au pays ou ailleurs dans le monde, ces femmes luttent contre les formes d'exclusion qu'elles perçoivent dans la société et qui se traduisent ici par la non disponibilité d'une offre dédiée à leur beauté. Elles luttent pour accéder à une forme de reconnaissance qui s'exprime par la consommation.

\section{Conclusion}

À Berlin comme à Paris, les pratiques de beauté se trouvent à la croisée d'enjeux individuels et sociaux pour les femmes noires et métissées. Interroger la présence d'une offre commerciale dédiée à ces beautés, c'est mettre en relief leur trop faible représentation dans les espaces publics de ces deux sociétés postmigratoires. C'est aussi montrer comment elles contournent et détournent les assignations et les frontières pour être pleinement reconnues comme membres de la société ; comment elles s'organisent par le biais de solidarités locales ou diasporiques pour accéder à ces produits et transformer les pratiques. Derrière la réponse à des besoins spécifiques, se joue ainsi bien plus que la conquête d'une beauté idéale. En recomposant quotidiennement des connexions multiples, familiales ou professionnelles, notamment avec le pays d'origine, mais aussi entre des capitales européennes comme Berlin et Paris, ces femmes participent activement au processus de circulation de biens matériels et symboliques. Grâce à elles, émergent de nouvelles manières d'être au monde et de nouveaux espaces culturels fondés sur du syncrétisme et de l'hybridité, facilités par l'émergence de médias globaux comme Internet qui transforment les sociétés européennes. 